\title{
Healthy Cities Indicators-A Suitable Instrument to Measure Health?
}

\author{
Premila Webster and Denise Sanderson
}

\begin{abstract}
The evidence-base for a health strategy should include information on the determinants of health and how they link together if it is to influence the health of the population. The WHO European Healthy Cities Network developed a set of 53 healthy city indicators (HCIs), to describe the health of its citizens and capture a range of local initiatives addressing the wider dimensions of health. This was the first systematic effort to collect and analyze a range of data from European cities. The analysis provided important insights into the interpretation, availability, and feasibility of collecting data, resulting in the development of a revised set of 32 indicators with improved definitions. An analysis of the revised indicators showed that this data was more complete and feasible to collect. It provided useful information to cities contributing to developing a description of health and thus helping to identify health problems. It also highlighted issues about the importance of collecting qualitative as well as quantitative data, the number of indicators and the appropriateness of using the indicators to compare different cities. HCIs facilitated the collection of routinely available health data in a systematic manner. The introduction of HCIs has encouraged cities to adopt a structured process of collecting information on the health of their citizens and build on this information by collecting appropriate local data for developing a city health profile to underpin a city health plan that would set out strategies and interventions to improve health and provide the evidence-base for health plans.
\end{abstract}

KEYWORDS Healthy cities, Health indicators

\section{CONTEXT}

The WHO European Healthy Cities Network (WHO-EHCN) encompasses a variety of people, institutions, cultures, and activities. The health of people living in towns and cities is strongly determined by their living and working conditions, the quality of the physical and socio-economic environment and the quality and accessibility of health care services. Healthy Cities is about changing the ways in which individuals, communities, private and voluntary organizations, and local governments think about, understand, and make decisions about health. Ultimately, Healthy Cities is about enhancing the physical, mental, social, and environmental well-being of the people who live and work in cities. ${ }^{1,2}$ Defining, describing, and measuring the "health" of a city is complex task. It needs to take account of the physical, psychological, emotional, and spiritual health of the citizens. Several factors interact

Webster is with the Department of Public Health, University of Oxford, Oxford, UK; Sanderson is with the National Institute of Public Health, Copenhagen, Denmark.

Correspondence: Premila Webster, Department of Public Health, University of Oxford, Oxford OX3 7LF, UK. (E-mail: premila.webster@dph.ox.ac.uk) 
to affect the health of individuals. They can be grouped into four main categories: health promotion, health services, social care, and environmental improvement (which includes physical, social, and economic environment). ${ }^{3}$

\section{Rationale Underpinning the Development of the Healthy City Indicators}

Health indicators are essentially numeric measures of health and well-being. Welldesigned and carefully selected indictors can help a community determine where it is, where it is going, and how far it is from chosen goals. ${ }^{4}$

The objectives of the collection and analysis of indicators of HCIs were to:

1. Provide a description of health in the city

2. Provide a baseline of information to make comparisons over time

3. Compare and contrast cities allowing for the differing socioeconomic and cultural differences between countries in the region

4. Identify associations between selected indicators

In order to provide a comprehensive picture of health, the indicators covered the areas of health, health services, environment, and socioeconomic conditions. The purpose of collecting such data was to facilitate more evidence-based, rational policy making and priority setting in relation to health. The information was also intended to inform the development of city health profiles (CHPs) and lay the foundations of specific city health plans to improve and sustain the health of citizens. Thus HCIs were a part of a logical sequence, beginning with collection of routinely available data in an attempt to identify those aspects of the city that contribute to or detract from the health of the population. This information could then be built on and expanded by collecting appropriate local data to develop a health profile of the city to stimulate political and administrative action in order to create healthy public policies based on evidence and ultimately action towards these policies.

\section{Development of the Initial HCls}

The need for HCIs to monitor progress, make comparisons within a city, and to stimulate change had been clear from the outset of the WHO-EHCN. However, devising indicators, which captured a range of local initiatives addressing the wider dimensions of health, and then using them across cities, proved to be challenging to WHO Healthy Cities Project Office. ${ }^{5}$ A working group was set up to meet these challenges and develop a set of indicators, to cover various aspects of city life. Fiftythree indicators covering the areas of "health" (including the traditional indices of mortality), health services, environment, and social and economic conditions ${ }^{6}$ were developed. All cities applying to join Phase II (1993-1997) of the WHO-EHCN were required to collect and submit the data (in the format of a structured questionnaire that defined and described each indicator) to the WHO-EHCN office. The analysis of this collection was published in $1999 .{ }^{6}$

Following the analysis, it was recommended that:

(1) A smaller core of the indicators should be developed from those identified as useful in the first collection

(2) Healthy Cities coordinators who would coordinate the data collection should be trained to enable them to undertake the task effectively and efficiently 


\section{Revised Indicators}

Network cities that had participated in the collection of the indicators reported that in spite of the problems of gathering and interpreting the data, the exercise was beneficial and served to strengthen intersectoral links within the city. ${ }^{9}$ It was therefore decided to develop a second set of indicators. This resulted in the review of the original set of indicators in $1998,{ }^{7}$ resulting in a more concise set of 32 indicators with improved definitions. ${ }^{8}$ They consisted of four groups (Table 1):

The analysis of the collection of the revised indicators was carried out towards the end of Phase III by the Danish National Institute of Public Health in Copenhagen. ${ }^{6}$ The data collected in the second round was more complete than the first round, with enough data available to carry out analysis of 31 out of the 32 indicators. The one indicator with insufficient data was on the "percentage of disabled persons employed." Of the 40 cities, 19 reported that the data was not available. ${ }^{6}$

\section{Lessons Learnt from the Collection and Analysis of the Revised HCls}

\section{Quantitative vs. qualitative data}

Data on the 32 indicators were requested primarily in a quantitative format but, as with the previous collection, many lent themselves to more qualitative responses and analysis. The three health indicators: all cause mortality, cause of death, and low birth rate are traditional health indicators with internationally recognized definitions and guidelines. Therefore, information relating to these indicators was available in the cities and relatively straightforward to analyze and interpret. Other quantitative indicators, primarily those relating to socioeconomic conditions, were also widely recognized, but lacked standard international definitions. This set of indicators is often culturally specific and highly dependent on specific policies adopted in the different countries on issues such as employment, social security, housing, and education. ${ }^{6}$

Several of the responses to the data collection on indicators bordered on the qualitative, in that the comments offered with the numbers proved more informative than the quantitative information. The qualitative information, which was extracted from the questionnaires, provided valuable insights into political and cultural approaches to the wider dimensions of health. ${ }^{6}$ Not surprisingly, these indicators also proved the most difficult for the cities to collect.

2. How many indicators?

Following the presentation of the results of the revised indicators to the member cities, there was interest in returning to the original 53 indicators, or at least a modified version, as it was thought that the current indicators did not provide a comprehensive view of health in the cities. While it is true that the revised indicators were not comprehensive, the data collected from revised set of 32 indicators were more complete than the original set of data collected and therefore lent itself to more accurate interpretation of the results. ${ }^{6}$

3. Is comparative analysis appropriate and valid?

One of the aims of collecting and analysing HCIs data was to look at trends over time. Due to changes in participating cities and the indicators themselves, a comparative analysis was possible on only nine indicators (two health, one health service, five environmental, and one socio-economic). However, the extent of 


\section{TABLE 1 Revised set of healthy city indicators}

\section{Health}

Mortality

Main causes of death

Low birth weight

2. Health services

City health education programs

Immunization rates

Inhabitants per primary health care practitioner

Inhabitants per nurse

Percentage of population covered by health insurance

Availability of services in foreign languages

Health debates in city council

3. Environmental indicators

Air pollution

Water quality

Sewage collection

Household waste treatment

Green space

Derelict industrial sites

Sport and leisure facilities

Pedestrianization

Cycle routes

Public transport access

Public transport range

Living space

4. Socioeconomic indicators

Percentage of population in inadequate housing

Homelessness

Unemployment

Poverty

Availability of child care

Age of mothers at time of birth

Abortion rate

Employment of disabled people

comparability varied between indicators, even comparisons within a particular city. The following issues were identified: ${ }^{6}$

A. Definition/interpretation-although comparisons were only undertaken on data collected from indicators for which the definition remained the same for the two collections, the way that an indicator is interpreted may be specific to a particular city and change over time.

B. Frequency of data collection - while cities are encouraged to include the most recent data available, this does not always mean that it was current. For example, while atmospheric pollution figures provided were current in the first collection, for the second collection the most recent figures available were 34 years old. For some indicators such a difference will not be that important, but for others (e.g. unemployment), the difference could be significant.

C. Population concerned-the cities were asked to specify the population the data referred to, e.g. part of the city, city, city and suburbs, or other. As the 
large majority of cities failed to provide this information, it was not taken into account in the analysis. Therefore, it is possible that differences between the two data collections could reflect different denominators.

D. Origin of data - the data provided by the cities comes from a variety of sources, including both public and private sector organizations. Thus, unless specifically stated, it was not always possible to identify whether both are included in the data collected. For example, data on the number of sports facilities may only be available from the public sector and thus exclude privately owned facilities. In addition, often, not one agency is responsible for collecting the data.

The following examples illustrate some of these issues ${ }^{6}$ :

\section{CAUSE OF DEATH}

The cause-specific death rates are crude rates per 100,000 total population in the cities. Over $60 \%$ of cities provided data on every cause of death except cancer of the buccal cavity, pharynx, and larynx, as well as AIDS, for which $58 \%$ and $45 \%$, respectively, provided data. From the raw data returned by the cities, the biggest contributor to cause of death across the cities was disease related to circulation (ICD9 390-459). Within this diagnostic grouping, ischaemic cardiomyopathies (ICD9 410-414) contributed almost twice as much to total mortality as vascular cerebral disease (ICD9 430-438). The next largest contributors to overall mortality were respiratory disease (ICD9 460-519), lung and bronchial cancer (ICD9 160$165)$, and trauma and intoxication. These results are illustrated in Figure 1.

The patterns of mortality from causes contributing to a smaller proportion of the overall mortality also raised interesting questions. There would appear to be a 59fold difference in mortality from cervical cancer (ICD9 180) between cities with the highest and lowest rates, although both cities are in southern Europe. There also appears to be a 62-fold difference in mortality from breast cancer (ICD9 174) between cities with the highest and lowest rates. The interpretation of such patterns,

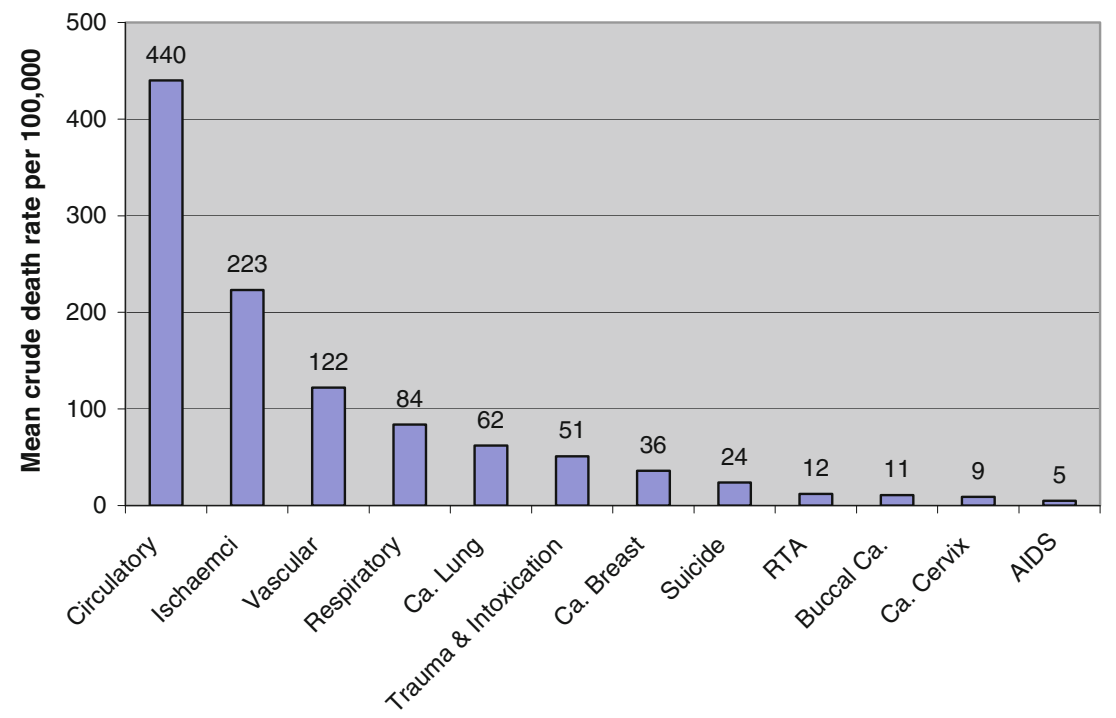

FIGURE 1. Major causes of mortality in the 'Healthy Cities'. 
however, is dependent on the validity of the data provided. In addition, the data is not standardized, so there are likely to be differences due to confounding factors, e. g. age.

\section{NUMBER OF HEALTH-RELATED QUESTIONS EXAMINED BY THE CITY COUNCIL EVERY YEAR}

This indicator seeks information about health-related questions raised by the elected representatives of the city, as well as the number of meetings organized dealing with matters of health.

Seventy-five percent of cities provided data, but there was wide variation in the frequencies recorded. Twenty of the cities $(87 \%)$ reported that 150 or fewer questions were examined yearly by the city council. The three cities with the highest reported values report 682 (Seixal), 924 (Turku) and a 1,750 (Stockholm). ${ }^{6}$ While differing levels of interest in health issues in the various city administrations probably exist, these results are likely to reflect more on the methods of capturing and defining the information.

\section{HOUSEHOLD WASTE TREATMENT QUALITY INDEX}

Ninety-four percent of the cities provided data on this indicator, giving information on the type and percentage of treatment used for household waste. Figure 2 indicates the different types of household waste treatment used by the cities.

Six cities reported treatment of household waste by methods that could not easily be included under the existing categories. Copenhagen (Denmark) reported that $0.1 \%$ was treated as hazardous waste; Brno (Czech Republic) reported that 7\% was illegal waste; Bologna and Milan (Italy) reported 33.4\% and 69\%, respectively, treated as discharge/ storage or "other treatments/controlled tips"; while Camden (London, UK) indicated that nearly all $(98.5 \%)$ of its waste went to an "engineered landfill." 6

\section{RELATIVE SURFACE AREA OF GREEN SPACES IN THE CITY}

Data was provided by all cities, however, the range varied enormously, ranging from $0.31 \%$ (Arezzo, Italy) to $61.53 \%$ (Gothenburg, Sweden). While differences in the size of green areas in the various cities undoubtedly exist, it is more likely that such a wide range of responses highlights the problem of definition. A number of cities included only public areas, while others took a broader interpretation of the term "green spaces" and included school/sports grounds and cemeteries ${ }^{6}$ (Table 2).

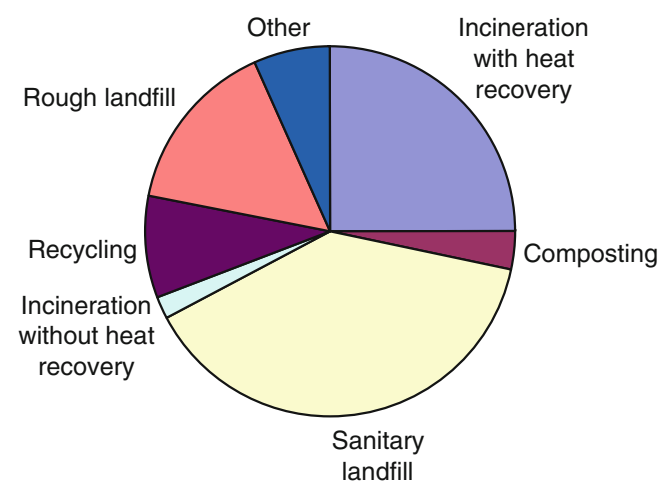

FIGURE 2. Household waste treatment in the 'Healthy Cities'. 
TABLE 2 Comments on the indicator "Relative area of green spaces in the city"

\begin{tabular}{|c|c|c|}
\hline City & Country & Comment \\
\hline Brno & Czech Republic & $\begin{array}{l}\text { The green spaces of the city include: urban green spaces, } \\
\text { including cemeteries, and landscape green spaces, } \\
\text { including forests }\end{array}$ \\
\hline Athens & Greece & $\begin{array}{l}\text { This value represents all open areas in public ownership } \\
\text { including hills, city squares, open-air sports facilities, } \\
\text { archaeological sites, courtyards in public buildings, etc. }\end{array}$ \\
\hline Zagreb & Croatia & Data does not include the Natural Park Medvednica \\
\hline Gyor & Hungary & There is no data available about gardens \\
\hline Dublin & Ireland & $\begin{array}{l}\text { Includes some lands, which are not "green spaces" but are } \\
\text { "open spaces" }\end{array}$ \\
\hline Geneva & Switzerland & $\begin{array}{l}\text { The data only includes green spaces open to the public, like } \\
\text { parks, graveyards, and public gardens managed by the city }\end{array}$ \\
\hline Liverpool & United Kingdom & $\begin{array}{l}\text { Total "green spaces" by our definition includes parks, golf } \\
\text { courses, farmland, cemeteries, and playing fields }\end{array}$ \\
\hline Stoke-on-Trent & United Kingdom & Figure includes countryside/Green Belt within city boundaries \\
\hline
\end{tabular}

\section{Views of Cities on the Availability, Relevance and "Inter-city Comparability Potential" of the Indicators}

An electronic questionnaire listing each of the $32 \mathrm{HCIs}$ was sent out to the cities. Information was requested on a scale from 0 to 5 regarding the following three criteria:

Availability of indicator data-despite some differences, data availability was not a significant problem for the cities. In general, information on the health indicators was easily available, while information on the health service and socioeconomic indicators appears to be more difficult to obtain.

Relevance for healthy city work - cities were of the opinion that all of the indicators were relevant, at least to some degree, in carrying out healthy city work locally. This is particularly true of those specifically relating to health and the physical environment.

Inter-city comparability - while it is very important for cities to have their own data for profiling and planning purposes, there is also much to be gained from comparing themselves with other cities. Cities were interested in these comparisons, particularly with regards the health indicators. Again, because these are fairly straightforward to analyze and interpret, they are also popular and easy to use. Cities also expressed an interest in comparing with other cities their "standing" on some of the environmental and socioeconomic indicators.

Six indicators appear in the top 10 of all three criteria. This means that data on them are easily available, are relevant to local Healthy Cities work, and cities are interested in using them for comparison. They include following two health indicators, three environmental indicators and one socioeconomic indicator:

1. Mortality—all causes

2. Cause of death

3. Atmospheric pollution

4. Cycling in the city

5. Public transport

6. Unemployment rate 


\section{DISCUSSION}

There are numerous international agencies, institutions, and other programs that use indicators to measure and monitor urban health. In an effort to provide an integrated and comprehensive view of the urban experience, it is important to coordinate knowledge and research in these areas. This will facilitate the harmonization of indicator development methods, indicator sets, and reporting standards, thus resulting in a more integrated option for monitoring urban health in Europe. A report entitled "Urban Health Indicators in Europe," ${ }^{10}$ presenting an overview of urban health indicator initiatives, made an effort to provide a comprehensive view of the urban experience. This exercise provided a unique opportunity to reflect on the HCIs.

The overall objective of collecting and analysing HCIs were very similar to those of the other European initiatives, i.e. to provide baseline information about a city, describe the determinants of health in the city, and allow comparison of these aspects. However, some very real differences exist when it comes to the type and number of indicators. While health, environment, and socioeconomic indicators of Healthy Cities are well established in initiatives throughout Europe, the same cannot be said of the health service indicators. The vast majority of these appear to be unique to the HCP. As regards the number of indicators, the total ranged from 300+ (Norway's Health Indicator System) to 10 (European Common Indicators Initiative).

The overall aim of collecting data on the indicators is to help identify those aspects of a city that contribute to or detract from the health of the population and observe how they change over time. Local policymakers can then use this information to make decisions as regards priority setting, resource allocation, and constructive solutions to urban problems. Data collected through HCIs have been used to inform the development of CHP and lay the foundations of city health plans. ${ }^{4}$ Combined with other health-related measures, an analysis of the information gained by the indicators could assist cities identify not only problems, but potential solutions as well. This, in turn, leads to the development of city health plans, which set out specific strategies and programs of interventions to improve health in the cities.

Data gathered from HCIs relating to mortality, morbidity, disability, environmental factors, uptake of preventative factors, etc. supply information needed to identify inequalities. What is important is that information is provided in a way that helps the city target resources towards those in greater need. To do this, the information needs to be linked to:

(1) Smaller geographical areas within the city (to identify communities needing specific support)

(2) Population groups known to be vulnerable, e.g. ethnic minorities, low income groups

If this is done, it will be possible to compare the health of groups of the population and identify where there are inequities. Collecting baseline information will also enable changes to be measured over time as a result of intervention programs. Thus the collection and appropriate analysis of HCIs could play a part in monitoring and evaluating the actions of the project towards improving inequity.

While work on the indicators of Healthy Cities highlights some of the complexities in collecting and analysing data at the European city level, it also illustrates the wealth of data available to measure and monitor health of people living in urban areas of Europe. However, there are still some questions that remain. 
Ideally, indicators should provide valid, reliable information that is relevant to policy makers and practitioners. Therefore, questions about which type of indicators are most appropriate should be made on the basis of what information is likely to provide the best possible data. Often, the "best" indicators depend to a large extent on the needs and circumstances of a given region or institution and reflect both the availability of data and local priorities. However, it is also important that indicators are not selected, simply because the data is readily available.

As regards the optimal number of indicators, this depends on many factors, including time and resources available for collection and the specific needs of the community. While the number of HCIs have been reduced from 53 to 32, there was much discussion among the cities as to whether this number is still too few or too many. While it is true that the current 32 indicators are not comprehensive and the cities could collect additional information about other facets influencing the health of the citizen, there is some concern regarding the difficulty in collecting such additional data and its subsequent analysis and interpretation. This reflects the ongoing dilemma between indicators that are relevant and those where the data is readily available.

Some cities have expressed interest in additional indicators. These included information on mental health/depression, infant mortality, life expectancy, medical attendance in hospitals, lifestyle/behavioural factors, immigrants/migration, TB cases and the monitoring of various environmental pollutants.

Indicators can be used to assess progress over time but change is slow and affected by many factors apart from Healthy Cities. Therefore, "intermediate indicators" that measure process or actions rather than outcomes could be developed. It was thought that "intermediate process indicators" might be a useful way for cities to demonstrate that they are moving forward towards long-term aspirations and goals. It could also be used by cities to interact with politicians and the public. For example, while reduction of mortality from lung cancer and chronic obstructive pulmonary disease is a long-term goal, "intermediate indicators" towards this goal could be smoking cessation rates, access and effective use of various smoking cessation interventions, etc.

\section{CONCLUSION}

It is important is that any future developments must be underpinned by the questions:

(1) What is the purpose of the indicator?

(2) How will this information help influence and improve health in cities?

(3) How feasible is the collection and analysis of the data collected?

The issues outlined in the collection and analysis of HCIs highlight the inherent complexities in collecting and analyzing data on health and its determinants to provide a meaningful evidence base to determine health policy and identify appropriate interventions to improve health at the city level. However, HCIs are useful instruments as they provide baseline description of health and thus identify health problems. In addition, they can aid in evaluation and assess progress-by analysing trends to identify whether the health of the city is going in the right direction and whether this direction is being maintained. Indicators have always played a crucial role in measuring and monitoring health status in Healthy Cities. By using the data to provide relevant information for policy makers and the public, 
through city health profiles, indicators help cities understand the way in which the urban environment influences population health, thus paving the way to evidencebased, rational policy making and priority setting in relation to health.

\section{REFERENCES}

1. Duhl LJ. The healthy city: its function and its future. Heal Promot. 1986; 1: 55-60.

2. Tsouros AD. The WHO healthy cities project: state of the art and future plans. Heal Promot. 1995; 10: 133-141.

3. Crown J. Healthy cities programmes: health profiles and indicators. In: Takano T, ed. Healthy cities and urban policy research. London: Routledge; 2003.

4. Hart M. Guide to sustainable community indicators. 2nd ed. North Andover, MA, Sustainable Measures; 1999.

5. WHO Healthy Cities Project Office. A Guide to Assessing Healthy Cities-WHO Healthy Cities Papers, No. 3. Copenhagen; 1988.

6. National Institute of Public Health, Denmark. Analysis of baseline healthy cities indicators. 2nd ed. Copenhagen: WHO Regional Office for Europe, 2001 (Centre for Urban Health, document 5027375 2001/16); 2001.

7. Webster P, Price C, eds. Healthy cities indicators: analysis of data from cities across Europe. Copenhagen: WHO Regional Office for Europe, (ICP/HCIT/94 01/PB04); 1996.

8. WHO Regional Office for Europe. WHO Healthy Cities-Revised baseline Healthy Cities Indicators. Centre for Urban Health; March1998.

9. Doyle YG, Tsouros AD, Cryer PC, Hedley S, Russell-Hodgson C. Practical lessons in using indicators of determinants of health across 47 European cities. Heal Promot Int. 1999; 14(4): 289-299.

10. WHO Regional Office for Europe. Urban health indicators in Europe. Copenhagen, (Centre for Urban Health, document 5027375 2001/18); 2001. 